\title{
Interactive comment on "Dramatic increase of reactive VOC emission from ships at berth after implementing the fuel switch policy in the Pearl River Delta Emission Control Area" by Zhenfeng Wu et al.
}

\section{Anonymous Referee \#1}

Received and published: 25 November 2019

Ship emissions as important sources of air pollution at the coastal cities have raised widespread attention and their emission characteristics have been consistently studied by many researchers. Wu et al. presents the changes of VOC emissions from ships at berth after implementing the fuel switch policy at the ECA. They find that the apparent increase of reactive species in the VOC emissions due to the strategy and their second formation potentials including $\mathrm{O} 3$ and SOA are also estimated. This study is well motivated for the effect evaluation of emission control strategies. However, despite the potential meaning of results from this study, the presentation of this study needs be 
improved to a large extent, especially for the writing. Major comments: 1. Description of VOCs. The author measured 68 VOC species used by GC-MS/FID, but the author is very chaotic for the description of VOC species in this manuscript, using the term NMHCs or VOCs in different sentences. Which one is the accurate expression? Generally, NMHC concentrations are determined by subtracting the amount of $\mathrm{CH} 4$ constituents from the THC measured by FID. The samples collected in canisters and analyzed by a preconcentrator coupled to GC-MSD/FID are speciated VOCs. Could the PEMS system measure THC and $\mathrm{CH} 4$ concentrations? 2. More information about sampling and analysis is needed, such as sampling flow, sampling time, sampling temperature, the auxiliary load, the devices used for conventional pollutants, the and the standard gas for VOC measurement. 3. As mentioned by the author, the fuel composition is a very important factor for VOC profiles, which is a possible reason for the different VOC compositions of the tested ships with the previous results. Then, is there apparent difference of VOC compositions for tested ships using four different fuels? What is the trendy of the VOC emissions when correlating the diesel composition? 4. More concise. Academic writing is a big question for this manuscript. There are many simple mistakes appeared in substantial sentences, which are mostly summarized in minor comments. Polishing the language is strongly suggested. 5. The unified expression. The author wrote several types of phases to express the implementation of the fuel switch policy, such as after implementing the fuel switch policy, after the new policy, after implementing the policy, after the fuel switch, and after the implementation of the fuel switch policy. Choose a suitable phrase for this expression.

Minor comments: 1 . Line 24 The unit of EF is not unitized, $\mathrm{mg} / \mathrm{kg}$ and $\mathrm{mg} \mathrm{kg}-1$. 2. Line 26 more rich. .. is it not richer? 3. Line 34 The number of PM2.5 should be subscripted. 4. Line 34 "may threatens"? It doesn't need the plural form for the term "threaten". 5. Line 46 ECAs? 6. Line 54 ship emissions? 7. Line 57 Is it suitable using the word "combat"? 8. Line 78 reveals? 9. Line 84 emissions from ships? 10. Line 99 Are all the pollutant emissions accounted for $40 \%$ ? 11. Line 113 What is a PM2.5 cutting head? Please give an accurate description. 12. Line 120 ls the mass selective detector MSD? 
What about the mass spectrometer detector? 13. Line 105 have already used? 14. Line 133 The EF of CO2 is calculated not determined. As follows not as following. 15. Line 136 Is the unit of Cf (gÂůkg-1)iijß 16. Line 140 concentration? 17. Line 151-153 Why is the explanation of VOC composition change placed in this section? 18. Line 161 The number of C6 should be subscripted. 19. Line 156 What is NMHCs? Is the measured VOC species? 20. Line 158 limited... 21. Line 160 The EFs of CO2... 22. Line 160 Is it right "before to"? 23. Line 159-163 It should give a summary rather than displaying the tested results of every ship. 24. Line 166 The term "that" should be "those". 25. Line 168 What is the carbonaceous aerosol? Does that mean OC and EC? 26. Line 169 "As shown" is the correct form, please revise all of the forms in this manuscript. 27. Line 172 the EFs of? 28. Line 176 by marine gasoline? 29. Line 178 TVOCs? Does TVOCs denote the measured VOC species? 30. Line 180 the emissions? 31. Line 191 NMHCs? 32. Line 199 and 214 individual NMHCs? 33. Line 206 the fuel switch? 34. Line 208 the only alkynes? 35. Line 212 "were" should be revised to "was". 36. Line 217 might played? 37. Line 217 their emission are? 38. Line 223 emission from shipiij§ 39. Line 230 Ozone Formation Potentials (OFPs) is? 40. Line 245 ship-emitted VOCs at berth. . 41. Line 247 Please give the literature for the calculated method of SOAFPs. 42 . Line 247 normalized secondary organic aerosol reactivity? 43. Line 250 Like Zhang et.al reported? 44. Line 258-260 What is the problem told by the SOAFP difference under the high NOx and low NOx conditions? 45. Line 256 This decline of RSOA? 50. Line 262 What is the NMHCs? 51. Line 266267 How about the comparison of Huang et al. results and this study results? 52. Line 268 What is the reason for the lower RSOA of Xiao's results? 53. Line 271 Ships? 54. Line 273 one the three? 55. Line 278 the EF of VOCiijßLine 281 the EF of NMHCs? Which one is right? 56 . Line 281 Why explained the unit of fuel-based EF here? 57. Line 287 are not affected ? 58. Table $2 \mathrm{~g} \mathrm{~kg}-1$ fuel? 59. Figure 5 before and IFSP? 60 . Figure 4 The figure needs add the standard error bar. IFSP is the first appearance. Spell out all acronyms on first use in the abstract and in the body of the article. 61 . Figure 2 diagrams? 
Interactive comment on Atmos. Chem. Phys. Discuss., https://doi.org/10.5194/acp-2019-897, 2019.
ACPD

Interactive

comment 